ORIGINAL

\title{
Effectiveness of self-exercise on elderly patients after hip fracture : A retrospective cohort study
}

\author{
Seiji Kasuga, ${ }^{a}$ RPT, Ryo Momosaki, ${ }^{b, c}$ MD, PhD, MPH, Kiyotaka Hasebe, ${ }^{a}$ RPT, Masashi Sawabe, ${ }^{\text {a }}$ RPT, \\ and Akira Sawaguchi, ${ }^{a}$ RPT
}

a Department of Rehabilitation, Teikyo University School of Medicine University Hospital, Mizonokuchi, ${ }^{b}$ Department of Rehabilitation Medicine, Teikyo University School of Medicine University Hospital, Mizonokuchi, ${ }^{\circ}$ Department of Rehabilitation Medicine, The Jikei University School of Medicine

\begin{abstract}
The purpose of this study was to clarify the impact of self-exercise for elderly patients in an acute hospital after hip fracture. This retrospective observational study used data from the Japan Rehabilitation Database spanning 2005-2015. This study identified in-hospital hip fracture patients admitted to an acute hospital. After applying exclusion criteria, 375 patients were eligible. The primary outcome was motor Functional Independence Measure (FIM) efficiency. Of the patients with hip fracture, $39 \%$ performed self-exercises. Patients who performed self-exercise had significantly higher motor FIM efficiency than those who did not (1.22 vs. $0.79 ; P<0.01)$. Multivariable regression analysis showed that motor FIM efficiency was significantly and positively correlated with self-exercise (coefficient, $0.25 ; 95 \%$ confidence interval, 0.13 to $0.43 ; P<0.01$ ). The data suggest that self-exercise is associated with good rehabilitation outcomes in hip fracture patients. J. Med. Invest. 66 : 178-181, February, 2019
\end{abstract}

Keywords : activities of daily living, exercise, hip fractures

\section{INTRODUCTION}

Hip fracture is the most common fracture in the elderly. The aging of populations in developed countries has led to a worldwide increase in the number of patients with hip fracture (1). Hip fracture is associated with a reduced ability to perform activities of daily living, and rehabilitation is an important aspect of care in these patients (2).

Self-exercise has been suggested to be useful for covering the shortage of rehabilitation time and could be an effective way to compensate for a shortage of formal therapy.

A previous study investigated the effectiveness of self-exercise for rehabilitation patients after acute stroke (3), and suggested that self-exercise programs contribute to improving their activities of daily living at discharge. However, the effectiveness of self-exercise or other treatments outside of formal therapy for hip fracture patients remains unclear. If self-exercise is effective for recovery in patients with hip fracture, self-exercise it would provide a practicable rehabilitation option.

This retrospective observational study aimed to clarify, using data collected from the Japan Rehabilitation Database, the impact of self-exercise on rehabilitation outcomes for elderly patients with hip fracture in an acute hospital.

\section{PATIENTS AND METHODS}

This study was approved by the institutional review board of the

Received for publication October 9, 2018 ; accepted January 23, 2019.

Address correspondence and reprint requests to Ryo Momosaki, MD, $\mathrm{PhD}, \mathrm{MPH}$, Department of Rehabilitation Medicine, Teikyo University School of Medicine University Hospital, Mizonokuchi, 5-1-1 Futako, Takatsu-ku, Kawasaki, Kanagawa, 213-8507, Japan and Fax : +81-44844-3201.
Japanese Association of Rehabilitation Medicine, which waived the requirement for informed patient consent because of the anonymous nature of the data.

The Japan Rehabilitation Database was developed with the financial support of the Ministry of Health, Labour and Welfare of Japan (4). Detailed clinical data were collected for rehabilitation inpatients discharged from participating hospitals beginning in 2005 . This database comprises only voluntary samples, not random samples. The database includes unique identifiers for the following data of patients with hip fracture : age and sex ; Functional Independence Measure (FIM) scores (ranging from 18 [totally dependent] to 126 [totally independent]) (5) ; length of stay ; days from injury onset ; type of fracture ; and performing self-exercise or not. Rehabilitation staff collected baseline data at the time of admission. Outcome variables were collected at discharge. These data were submitted to the Japan Association of Rehabilitation Database, which extracted the data and sent it to researchers. By 2013, 78 hospitals had contributed structured data for 29,339 patients to the database. All personal data are coded, and any personally identifiable information has been deleted.

This study used data sourced from the Japan Rehabilitation Database on patients admitted between August 2005 and September 2015 with a diagnosis of hip fracture. We included only hospitals with information about self-exercise. Inclusion criteria for patients were 65 years or older, admitted no later than the day after injury, and information on FIM available at admission and discharge.

Rehabilitation programs for hip fracture focused on gait and exercise related to activities of daily living. The typical gym exercise program was composed of 20-40 minutes of physical therapy per day, on weekdays. This program included muscle-strengthening exercise, standing training, balance training, and walking (6).

Self-exercise programs varied in terms of content and load. Although the details were not clear for every facility, a survey of some of the facilities indicated that self-exercise programs were 
planned under the guidance of a therapist and that they primarily focused on standing training, transfer training, and gait training. Self-exercise programs should be performed with the instructions of a therapist, nurse, or physician to prevent accidental injury. Selfexercise programs therefore supplemented formal therapy by repeating the activity or motion.

We extracted the following data from the database : age, sex, FIM score at admission and discharge, days from injury, type of fracture, whether surgery had been performed, days from injury to surgery, and comorbidities (cerebrovascular disease and orthopedic disease) from medical charts. The FIM score, a basic indicator of disability severity, is widely used in Japanese rehabilitation hospitals and familiar to almost all rehabilitation staff. The instrument consists of 18 items, each of which is scored on a 7-point ordinal scale ; the higher the score for an item, the more independently the patient is able to perform the task required by that item. The FIM can be subdivided into a 13 -item motor subscale (eating, grooming, bathing, upper-body dressing, lower-body dressing, personal hygiene, bladder management, bowel management, bedto - chair transfer, toilet transfer, shower transfer, walk or wheelchair, and stairs) and a 5-item cognitive subscale (comprehension, expression, social interaction, problem solving, and memory). The motor subscale score ranges from 13 to 91 (motor FIM). The Japanese national health insurance scheme uses the bedridden degree to judge the level of long-term care (7). Motor FIM indicates the level of independence in daily living activities of elderly patients and ranges from independent to completely bedridden, as follows : independent (fully independent), J1 and J2 (independent with some disability), A1 and A2 (moving around indoors independently but needing some assistance when they go out), B1 and B2 (mostly bedridden), or C1 and C2 (completely bedridden). In this study, we divided this range of independence into four groups : independent (independent, J1 or J2), homebound (A1 or A2), mostly bedridden (B1 or B2), or completely bedridden (C1 or $\mathrm{C} 2)$.

The primary outcome was motor FIM efficiency, which was calculated as (discharge motor FIM score-admission motor FIM score) / (length of stay in days) (8). The secondary outcomes were motor FIM gain, motor FIM effectiveness, and discharge motor FIM score. Motor FIM gain was calculated as (discharge motor FIM score-admission motor FIM score). Motor FIM effectiveness was calculated as (motor FIM gain) / (91-admission FIM score) (8). Improvement of FIM is affected by length of stay, and FIM efficiency include length of stay in the formula. Because we believe FIM efficiency is suitable for the primary outcome.

The clinical characteristics and outcomes of the patients were compared between the patients who performed self-exercise (selfexercise group) and those who did not (control group) by the chisquare test for categorical variables and by the unpaired $t$ test for continuous variables.

Multivariable linear regression analysis for outcomes as the dependent variable was performed to calculate the correlation coefficients of the following independent variables : age, sex, type of fracture, comorbidities (cerebrovascular disease and orthopedic disease), pre-injury bedridden degree, admission motor FIM score, admission cognitive FIM score, amount of physical therapy, days from injury to surgery, and self-exercise (or control group). Cognitive function affects trainability for physical performance in exercise intervention among older adults with cognitive impairment (9). We suggested that patients with low cognitive FIM score might have difficulty with self-exercise. Therefore, we added admission cognitive FIM score as independent variable. We used SPSS 19.0 software (IBM SPSS Inc., Armonk, NY, USA) for all analyses. The threshold for significance was $P<0.05$.

\section{RESULTS}

A total of 838 hip fracture patients from 11 acute hospitals with information about self-exercise were identified during the study period. Of these, this study excluded 256 patients without admission until next day from injury, 37 patients less than 65 years old, 51 patients who were completely bedridden before admission, 36 patients who were non-operative and 83 patients without complete data about FIM. As a result, there were 375 patients in six acute hospitals eligible for analysis (Figure 1).

Table 1 shows the clinical characteristics of the study patients. The self-exercise group comprised 146 patients (38.9\%). The selfexercise group was significantly younger than the control group, and the admission motor and cognitive FIM score and daily time undergoing physical therapy were significantly higher in patients in the self-exercise group than in the control group. Times from injury to surgery were significantly shorter for patients in the selfexercise group than in the control group. There were also significant differences in the type of fracture and pre-injury bedridden degree between the two groups.

Table 2 shows the results of a comparison of outcomes between the two groups. The self-exercise group had significantly higher scores than the control group in motor FIM efficiency (mean \pm standard deviation, $1.22 \pm 0.73$ vs. $0.79 \pm 0.63$, respectively ; $P<$ $0.01)$, motor FIM gain ( $34.9 \pm 14.8$ vs. $25.2 \pm 16.7$, respectively ; $P<$ $0.01)$, motor FIM effectiveness $(0.63 \pm 0.24$ vs. $0.39 \pm 0.26$, respectively ; $P<0.01)$ and discharge motor FIM score (68.6 \pm 18.0 vs. $51.0 \pm 19.4$, respectively ; $P<0.01$.

Table 3 shows the multivariable linear regression analysis result for motor FIM efficiency. Multivariable analysis identified that selfexercise was significantly associated with higher motor FIM efficiency (coefficient, $0.25 ; 95 \% \mathrm{CI}, 0.13$ to $0.43 ; P<0.01$ ). Other independent variables that were significantly associated with motor FIM efficiency were age, sex, cerebrovascular disease, and preinjury independent. Additionally, as shown in Table 4, self-exercise was associated significantly with higher scores in motor FIM effectiveness (coefficient, $0.06 ; 95 \% \mathrm{CI}, 0.01$ to $0.12 ; P=0.01$ ). However, we could not find significant associations for motor FIM gain or discharge motor FIM score.

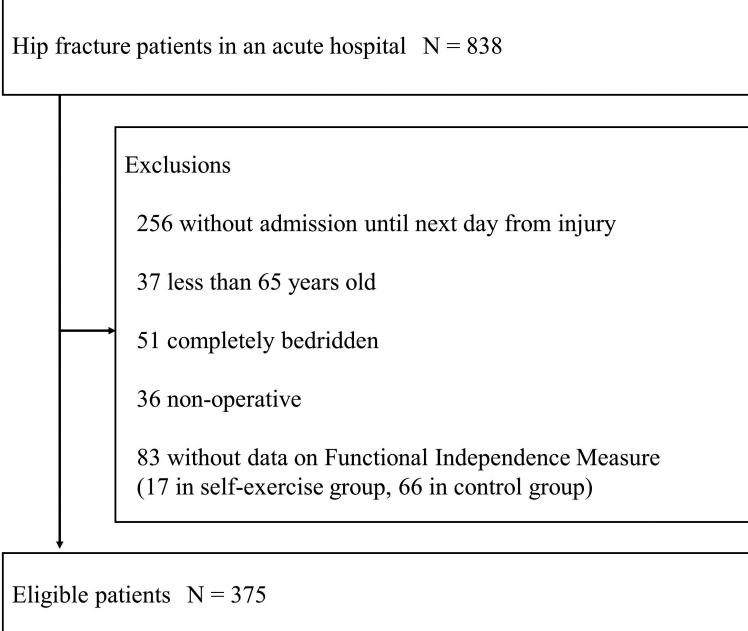

Figure 1. Flow chart of patient selection 
Table 1. Patients' characteristics

\begin{tabular}{|c|c|c|c|c|}
\hline & Total $(\mathrm{n}=375)$ & $\begin{array}{l}\text { Self-exercise group } \\
\qquad(\mathrm{n}=146)\end{array}$ & $\begin{array}{l}\text { Control group } \\
(\mathrm{n}=229)\end{array}$ & $P$ value \\
\hline Mean age \pm SD (years) & $84.5 \pm 7.6$ & $82.7 \pm 8.3$ & $85.6 \pm 6.9$ & $<0.01$ \\
\hline Female (\%) & $312(83.2)$ & $123(84.2)$ & $189(82.5)$ & 0.77 \\
\hline \multicolumn{5}{|l|}{ Fracture type (\%) } \\
\hline Femoral neck & $159(42.4)$ & $72(49.3)$ & $87(38.0)$ & \multirow[t]{3}{*}{$<0.01$} \\
\hline Trochanteric & $183(48.8)$ & $70(47.9)$ & $113(49.3)$ & \\
\hline Other & $33(8.8)$ & $4(2.7)$ & $29(12.7)$ & \\
\hline \multicolumn{5}{|l|}{ Comorbidities (\%) } \\
\hline Cerebrovascular disease & $47(12.5)$ & $20(13.7)$ & $27(11.8)$ & 0.70 \\
\hline Orthopedic disease & $82(21.9)$ & $38(26.0)$ & $44(19.2)$ & 0.12 \\
\hline \multicolumn{5}{|l|}{ Pre-injury bedridden degree (\%) } \\
\hline Independent & $150(40.0)$ & $92(63.0)$ & $58(25.3)$ & \multirow[t]{4}{*}{$<0.01$} \\
\hline Homebound & $125(33.3)$ & $38(26.0)$ & $87(38.0)$ & \\
\hline Mostly bedridden & $76(20.3)$ & $12(8.2)$ & $64(27.9)$ & \\
\hline Unknown & $24(6.4)$ & $4(2.7)$ & $20(8.7)$ & \\
\hline Admission motor FIM score \pm SD & $28.9 \pm 13.7$ & $33.8 \pm 14.7$ & $25.8 \pm 12.1$ & $<0.01$ \\
\hline Admission cognitive FIM score \pm SD & $21.8 \pm 9.7$ & $28.3 \pm 7.1$ & $17.7 \pm 8.8$ & $<0.01$ \\
\hline Amount of physical therapy (min/day) $\pm \mathrm{SD}$ & $36.0 \pm 12.2$ & $40.4 \pm 10.6$ & $33.1 \pm 12.3$ & $<0.01$ \\
\hline Length of stay (day) $\pm \mathrm{SD}$ & $39.7 \pm 27.5$ & $36.1 \pm 21.8$ & $41.9 \pm 30.5$ & 0.05 \\
\hline Rehabilitation starting from admission (day) $\pm \mathrm{SD}$ & $1.7 \pm 2.7$ & $1.3 \pm 2.1$ & $2.0 \pm 3.0$ & $<0.01$ \\
\hline Time from injury to surgery (day) \pm SD & $4.4 \pm 11.2$ & $3.0 \pm 14.7$ & $5.4 \pm 8.0$ & 0.04 \\
\hline
\end{tabular}

FIM, Functional Independence Measure; SD, standard deviation.

Table2. Outcomes of the self-exercise group and control group

\begin{tabular}{lcccc}
\hline & Total $(\mathrm{n}=375)$ & $\begin{array}{c}\text { Self-exercise group } \\
(\mathrm{n}=146)\end{array}$ & $\begin{array}{c}\text { Control group } \\
(\mathrm{n}=229)\end{array}$ & $P$ value \\
\hline Motor FIM efficiency \pm SD & $0.96 \pm 0.70$ & $1.22 \pm 0.73$ & $0.79 \pm 0.63$ & $<0.01$ \\
Motor FIM gain \pm SD & $28.9 \pm 16.6$ & $34.9 \pm 14.8$ & $25.2 \pm 16.7$ & $<0.01$ \\
Motor FIM effectiveness \pm SD & $0.49 \pm 0.28$ & $0.63 \pm 0.24$ & $0.39 \pm 0.26$ & $<0.01$ \\
Discharge motor FIM score \pm SD & $57.8 \pm 20.7$ & $68.6 \pm 18.0$ & $51.0 \pm 19.4$ & $<0.01$ \\
\hline
\end{tabular}

FIM, Functional Independence Measure ; SD, standard deviation.

Table 3. Multivariable linear regression analysis for motor FIM efficiency

\begin{tabular}{lccr}
\hline & B & $95 \%$ CI (B) & $P$ value \\
\hline Self-exercise & 0.25 & 0.13 to 0.43 & $<0.01$ \\
Age & -0.01 & -0.02 to -0.008 & 0.03 \\
Female sex & 0.26 & 0.05 to 0.45 & 0.01 \\
Femoral neck fracture & -0.02 & -0.13 to 0.08 & 0.44 \\
Cerebrovascular disease & -0.31 & -0.44 to -0.01 & 0.04 \\
Orthopedic disease & -0.05 & -0.21 to 0.12 & 0.38 \\
Admission motor FIM & -0.31 & -0.51 to -0.16 & $<0.01$ \\
Admission cognitive FIM & -0.30 & -0.48 to -0.11 & $<0.01$ \\
Pre-injury independent & 0.21 & 0.14 to 0.33 & $<0.01$ \\
Amount of physical therapy & 0.006 & -0.003 to 0.009 & 0.55 \\
Time from injury to surgery & -0.001 & -0.008 to 0.004 & 0.83 \\
\hline
\end{tabular}

FIM, Functional Independence Measure ; B, partial regression coefficient ; CI, confidence interval.

Table 4. Multivariable linear regression analyses for secondly outcomes

\begin{tabular}{lccc}
\hline & $\mathrm{B}$ & $95 \% \mathrm{CI}$ (B) & $P$ value \\
\hline Motor FIM effectiveness & 0.06 & 0.01 to 0.12 & 0.01 \\
Motor FIM gain & 2.43 & -1.36 to 6.22 & 0.21 \\
Discharge motor FIM score & 3.49 & -0.38 to 7.35 & 0.08 \\
\hline
\end{tabular}

FIM, Functional Independence Measure ; B, partial regression coefficient ; CI, confidence interval. 


\section{DISCUSSION}

In the present study, we used a large database of rehabilitation inpatients to examine the impact of self-exercise on rehabilitation outcomes in acute hip fracture patients. Self-exercise was significantly associated with increased motor FIM efficiency, motor FIM gain, motor FIM effectiveness, and discharge motor FIM score.

There are several reports which identified the important role of self-exercise $(10,11)$. In the field of rehabilitation, one study showed the effects of additional self-exercise program on activities of daily living in patients after acute stroke (3). In the present study, we investigated the effects of self-exercise in patients with hip fracture.

We showed that the self-exercise group had significantly higher motor FIM effectiveness than the control group. However, multivariable linear regression analysis did not find any significant association between self-exercise and motor FIM gain or discharge motor FIM score. The reason may be that FIM effectiveness is less affected by a ceiling effect than other outcomes.

Some researchers have shown the effect of large amount of exercise on muscle strength, functional performance, and functional ability in hip fracture patients $(12,13)$. However, many acute hospitals cannot provide enough rehabilitation to hip fracture patients due to a shortage of physical therapists. Self-exercise could be conducted without direct supervision by therapists. Self-exercise compensated for a shortage of formal therapy and it probably contributed to improvement of FIM without increasing cost.

Several limitations of this study should be acknowledged. First, the database that we used lacks detailed information about type of self-exercise. We could not investigate what type of self-exercise was appropriate for hip fracture patients. Second, the Japan Rehabilitation Database consists only of voluntary hospital samples, not random samples. As a result, application of these findings to all hip fracture patients undergoing rehabilitation may be limited. Third, we could not obtain enough information about type of surgery or type of dementia. However, when we adjusted for type of fracture, the influence of type of surgery was accounted for to some extent. Fourth, the database did not include information about the waiting period for transfer to convalescent rehabilitation hospitals. This missing data could influence motor FIM efficiency. Therefore, we added motor FIM gain, motor FIM effectiveness, and discharge motor FIM score as secondary outcomes to assess the robustness of our results.

In conclusion, self-exercise was associated with improved recovery of hip fracture in acute hospital. Self-exercise is probably be a feasible option to improve the quality of rehabilitative management.

\section{CONFLICT OF INTERESTS}

No conflict of interest to declare.

\section{ACKNOWLEDGMENTS}

This study gratefully acknowledges the Japan Association of Rehabilitation Database for its development of the Japan Rehabilitation Database, which was used in this study. This study's results represent the points of view of the authors, not the official views of the Japan Association for Rehabilitation Database.

\section{REFERENCES}

1. Johnell O, Kanis JA : An estimate of the worldwide prevalence and disability associated with osteoporotic fractures. Osteoporos Int $17:$ 1726-1733, 2006

2. Radosavljevic N, Nikolic D, Lazovic M, Jeremic A : Hip fractures in a geriatric population-rehabilitation based on patient's needs. Aging Dis $5: 177-182,2014$

3. Shiraishi N, Suzuki Y, Matsumoto D, Jeong S, Sugiyama M, Kondo K: Effects of a self-exercise program on activities of daily living in patients after acute stroke : a propensity score analysis based on the Japan Association of Rehabilitation Database. Arch Phys Med Rehabil 98 : 434-441, 2017

4. Inoue $\mathrm{Y}$, Jeong $\mathrm{S}$, Kondo $\mathrm{K}$ : Changes in hospital performance after implementation of pay-for-performance in Japan. Clinical Audit $3: 17-23,2011$

5. Hetherington H, Earlam RJ : Measurement of disability after multiple injuries : the functional independence measure. Clinical review. Eur J Surg 161 : 549-555, 1995

6. Chudyk AM, Jutai JW, Petrella RJ, Speechley M : Systematic review of hip fracture rehabilitation practices in the elderly. Arch Phys Med Rehabil 90 : 246-262, 2009

7. Arai Y, Zarit SH, Kumamoto K, Takeda A : Are there inequities in the assessment of dementia under Japan's LTC insurance system? Int J Geriatr Psychiatry $18: 346-352,2003$

8. Koh GC, Chen CH, Petrella R, Thind A : Rehabilitation impact indices and their independent predictors : a systematic review. BMJ Open 3 : e003483, 2013

9. Uemura K, Shimada H, Makizako H, Doi T, Yoshida D, Tsutsumimoto K, Anan Y, Suzuki T: Cognitive function affects trainability for physical performance in exercise intervention among older adults with mild cognitive impairment. Clin Interv Aging 8:97-102, 2013

10. Kim M, Lee M, Kim Y, Oh S, Lee D, Yoon B : Myofascial Pain Syndrome in the Elderly and Self-Exercise : A Single-Blind, Randomized, Controlled Trial. J Altern Complement Med 22 : 244-251, 2016

11. Kim C, Youn JE, Choi HE : The effect of a self exercise program in cardiac rehabilitation for patients with coronary artery disease. Ann Rehabil Med 35 : 381-387, 2011

12. Hauer K, Specht N, Schuler M, Bärtsch P, Oster P : Intensive physical training in geriatric patients after severe falls and hip surgery. Age Ageing 31 : 49-57, 2002

13. Tokunaga M, Kondo K : Relationship between Training Time and Motor FIM at Discharge in Patients with Femoral Neck Fracture. Jpn J Rehabil Med 52 : 751-759, 2015 